\title{
The Casimir effect from a Condensed Matter Perspective
}

\author{
L. Pálová, P. Chandra, and P. Coleman \\ Center for Materials Theory, Department of Physics and Astronomy, \\ Rutgers University, Piscataway, New Jersey 08854
}

\begin{abstract}
The Casimir effect, a key observable realization of vacuum fluctuations, is usually taught in graduate courses on quantum field theory. The growing importance of Casimir forces in microelectromechanical systems motivates this subject as a topic for graduate many-body physics courses. To this end, we revisit the Casimir effect using methods common in condensed matter physics. We recover previously derived results and explore the implications of the analogies implicit in this treatment.
\end{abstract}




\section{INTRODUCTION}

The Casimir effect results from the interplay of zero-point fluctuations and boundary conditions, and leads to the attraction between two parallel conducting plates in a vacuum. ${ }^{1,2,3}$ It was one of the first predicted, observable consequences of vacuum fluctuations. Traditionally it has been taught in graduate courses on quantum field theory $\stackrel{4}{*}$ Because the Casimir force scales inversely proportionally to the fourth power of the plate separation $a$, it is only measurable when $a$ is quite small (micron regime). Recently, the Casimir phenomenon has assumed a new importance in the design of nanoscale devices., effect to critical systems with external constraints continue to be fruitful.11,12,13,14,15,16 It therefore is timely to include it in graduate courses on condensed matter theory.

In this paper we revisit the Casimir effect, recovering previously derived results $1,17,18,19,20,21$ with an approach used to calculate observable properties in finitetemperature solids, and can therefore be naturally included in the many-body curriculum, ${ }^{22}$ possibly serving as a simple pedagogical example of these techniques.

\section{THE CASIMIR COEFFICIENT}

The Casimir effect results from the effect of boundary conditions on the zero-point fluctuation modes of the electromagnetic field. We will consider the simplest case of two parallel conducting plates. The energy, $\Delta \mathcal{E}$, is the finite difference between the zero-point energies with and without the plates, $, 117,18,19,20$ and the force is then the spatial derivative of $\Delta \mathcal{E}$. The component of the electric field parallel to the conducting plates must vanish. There are two sets of modes that satisfy this condition: the transverse electric (TE) and transverse magnetic (TM) modes where the electric or magnetic field are respectively parallel to the plates. ${ }^{23}$ The electric field for the transverse electric field modes is given by

$$
\vec{E}^{\mathrm{TE}}(\vec{x}, z)=\sum_{\vec{q}_{\perp}, n>0} E_{\vec{q} n}\left(\hat{z} \times \hat{q}_{\perp}\right) e^{i \vec{q}_{\perp} \cdot \vec{x}} \sin \left(\frac{n \pi}{a} z\right), \quad(n>0)
$$

where $\vec{x}$ and $z$ are the co-ordinates parallel and perpendicular to the plates respectively, $n$ is an integer, and $E_{\vec{q} n}$ is the Fourier amplitude of the fields. There is no $n=0$ TE mode. The corresponding magnetic field is calculated using Faraday's equations $\vec{\nabla} \times \vec{E}=-\partial \vec{B} / \partial t$, or 


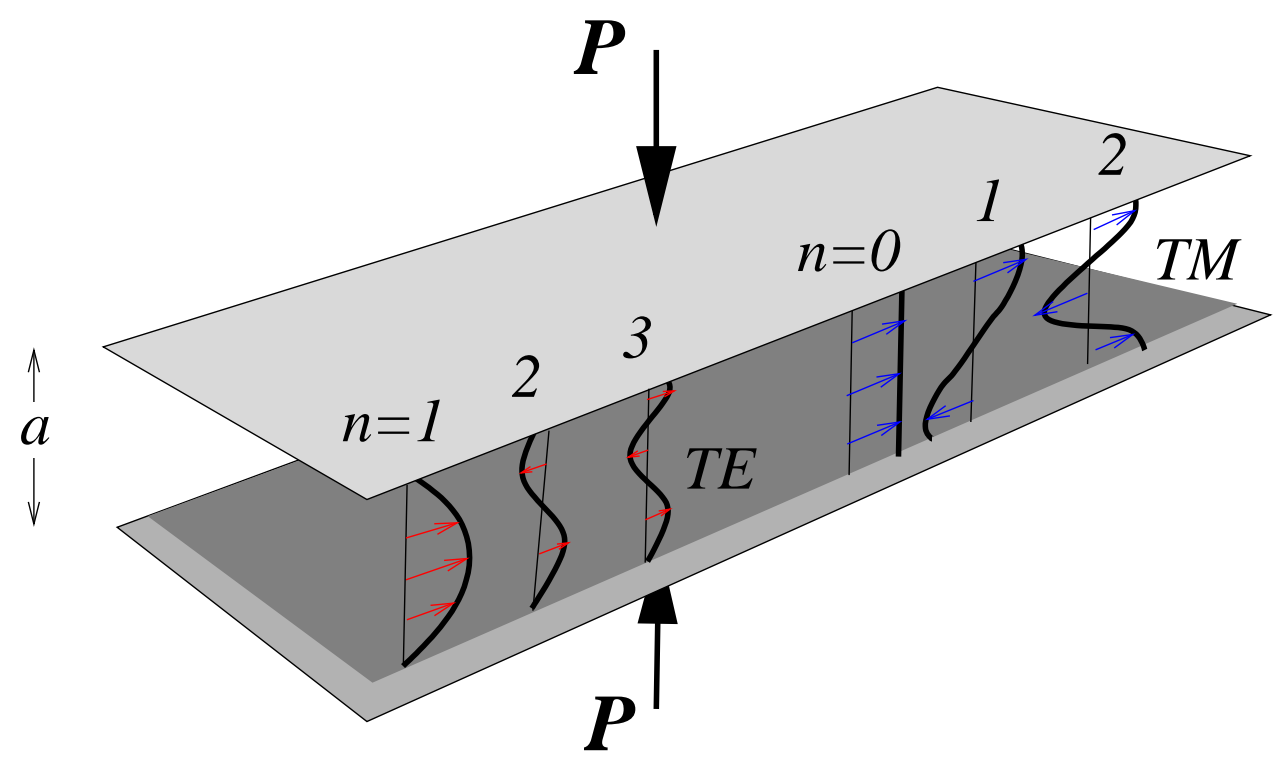

FIG. 1: Schematic of the Casimir effect indicating that the normal modes of the electromagnetic field between two conducting plates occupy a discrete set of wavevectors. In the transverse electric (TE) modes the electric field lies parallel to the plates and $n \geq 1$. In the TM modes the magnetic field lies parallel to the plates and $n \geq 0$. The modification of the frequencies of the zero-point fluctuations by boundary conditions changes the energy of the system, creating a pressure on the plates.

$\vec{B}=\frac{1}{i \omega} \vec{\nabla} \times \vec{E}$. The magnetic field for the TM field modes is given by

$$
\vec{B}^{\mathrm{TM}}(\vec{x}, z)=\sum_{\vec{q}_{\perp}, n>0} B_{\vec{q} n}\left(\hat{z} \times \hat{q}_{\perp}\right) e^{i \vec{q}_{\perp} \cdot \vec{x}} \cos \left(\frac{n \pi}{a} z\right), \quad(n \geq 0)
$$

We note that there is one extra $n=0$ TM mode. The corresponding electric fields are computed from Maxwell's displacement current equation $\vec{\nabla} \times \vec{B}=\frac{1}{c^{2}} \frac{\partial \vec{E}}{\partial t}$ or $\vec{E}=-\frac{c^{2}}{i \omega}(\vec{\nabla} \times$ $\vec{B})$. The Fourier modes of these fluctuations thus involve a discrete set of wavevectors,

$$
\vec{q}_{n}=\left(\vec{q}_{\perp}, q_{z n}\right)
$$

where $q_{z n}=n \pi / a$ and $n$ is an integer, leading to a discrete set of normal mode frequencies $\omega_{\vec{q}_{\perp} n}=c \sqrt{\vec{q}_{\perp}^{2}+q_{z n}^{2}}$ (see Fig. 1), where $c$ is the speed of light.

The zero-point energy of the fields inside the plates is given by

$$
\mathcal{E}_{C}=\sum_{\vec{q}_{\perp}} \frac{\hbar \omega_{\vec{q}_{\perp}, 0}}{2}+2 \sum_{\vec{q}_{\perp}, n>0} \frac{\hbar \omega_{\vec{q}_{\perp}, n}}{2}
$$


where the first term is the zero point energy of the $n=0$ TM mode, and the second term counts the zero point energy of the TM and TE modes with $n>0$. We may rewrite these two terms as a sum over all $n$, both positive and negative, as follows

$$
\mathcal{E}_{C}=\frac{\hbar c}{2} \sum_{n=-\infty}^{\infty} \sum_{\vec{q}_{\perp}} \sqrt{q_{\perp}^{2}+q_{z n}^{2}} .
$$

In the continuum limit we let $\sum_{\vec{q}_{\perp}} \rightarrow A \int \frac{d^{2} q_{\perp}}{(2 \pi)^{2}}$, where $A$ is the area of the plates, to obtain

$$
\mathcal{E}_{C}=A \frac{\hbar c}{2} \sum_{n=-\infty}^{\infty} \int \frac{d^{2} q_{\perp}}{(2 \pi)^{2}} \sqrt{q_{\perp}^{2}+q_{z n}^{2}} .
$$

The quantity $\mathcal{E}_{C} / A$ determined from Eq. ([6) is dimensionally of the form $\left[\mathcal{E}_{C} / A\right]=\hbar c\left[L^{-3}\right]$. Because $a$ is the only length scale in the system, it follows that the change in the zero-point energy must have the form

$$
\frac{\Delta \mathcal{E}_{c}}{A}=\mathcal{K} \frac{\hbar c}{a^{3}}
$$

The fact that this Casimir energy is sensitive to arbitrary interplate separation, $a$, is a direct consequence of the gaplessness, and thus the scale-free nature of the photon field.

The traditional calculation of $\mathcal{K}$ in the Casimir energy Eq. (17) is performed using a regularization procedure enforced by a zeta function. $1,17,19$ In this paper we present an alternative derivation in which we calculate the necessary sums by exploiting the structure of the Bose function and the residue theorem of complex analysis. This approach is central to the Mat-

subara formalism ${ }^{22}$ used to study many-body systems at finite temperature. The calculation of $\mathcal{K}$ by contour integration has been discussed,,$\underline{18,20}$ and we will adapt this treatment as an example of the Matsubara method 22 in a graduate course in many-body physics. Therefore we take a brief diversion to describe the technique in general before applying it specifically to the calculation of the Casimir coefficient.

\section{THE MATSUBARA APPROACH}

We begin by noting that the Bose function

$$
n_{B}(z)=\frac{1}{e^{\hbar z / k_{B} T}-1}
$$

has poles on the imaginary axis (see Fig. 2) at $z=i \nu_{n}$, where $\nu_{n}=n 2 \pi k_{B} T / \hbar$, because

$$
e^{i \hbar \nu_{n} / k_{B} T}=e^{2 \pi n i}=1
$$


Next we take

$$
z=i \nu_{n}+\delta
$$

where $\delta$ is small so that $z$ is slightly off the imaginary axis so that

$$
n_{B}\left(i \nu_{z}+\delta\right)=\frac{1}{e^{\hbar \delta / k_{B} T}-1} \approx \frac{k_{B} T}{\hbar \delta},
$$

from which we see that $k_{B} T$ is the residue at each of the poles $z=i \nu_{n}$ of $n_{B}(z)$, so that

$$
n_{B}(z)=\sum_{n} \frac{k_{B} T}{\hbar\left(z-i \nu_{n}\right)} .
$$

If we have a function, $F(z)$, that does not have poles on the imaginary axis, we can use the residue theorem and Eq. (12) to write

$$
\oint_{\mathcal{C}} d z F(z) n_{B}(z)=2 \pi i \sum_{n} \frac{k_{B} T}{\hbar} F\left(i \nu_{n}\right)
$$

where $\mathcal{C}$ is a contour that encircles the imaginary axis in a clockwise sense, as shown in Fig. 2, Equation (13) can be rearranged to read

$$
\sum_{n} F\left(i \nu_{n}\right)=\frac{\hbar}{k_{B} T} \oint_{\mathcal{C}} \frac{d z}{2 \pi i} F(z) n_{B}(z)
$$

which is a key result in the Matsubara approach used to evaluate sums that emerge in the study of many-body systems at finite temperatures $\underline{\underline{22}}$

We will now apply Eq. (14) to the specific case of the Casimir coefficient. To do so, we must identify the summations that we need to calculate. We begin with the zero-point energy per unit area (6)

$$
\frac{\mathcal{E}_{C}}{A}=\frac{\hbar c}{2} \sum_{n} \int \frac{d^{2} q_{\perp}}{(2 \pi)^{2}} \sqrt{q_{\perp}^{2}+q_{z n}^{2}}
$$

We are interested only in the change in the zero point energy as a result of the plates. In the limit of infinite plate separation $(a \rightarrow \infty)$ the discrete interval in $q_{z n}, \Delta q_{z n}=\pi / a$, becomes infinitesimal, and the sum over $n$ in Eq. (15) can be replaced by an integral $\sum_{n}=$ $\sum_{n} \frac{\Delta q}{\pi / a}=\frac{a}{\pi} \int d q_{z}$. Therefore the change in the zero-point energy per unit area due to the presence of the plates is given by

$$
\frac{\Delta \mathcal{E}_{C}}{A}=\frac{\mathcal{E}_{C}}{A}-\left.\frac{\mathcal{E}_{C}}{A}\right|_{a \rightarrow \infty}=\hbar c a \int \frac{d^{2} q_{\perp}}{(2 \pi)^{2}} I\left(q_{\perp}, a\right),
$$

where

$$
I\left(q_{\perp}, a\right)=\frac{1}{2 a} \sum_{n} \sqrt{q_{\perp}^{2}+q_{z n}^{2}}-\int \frac{d q_{z}}{2 \pi} \sqrt{q_{\perp}^{2}+q_{z}^{2}} .
$$




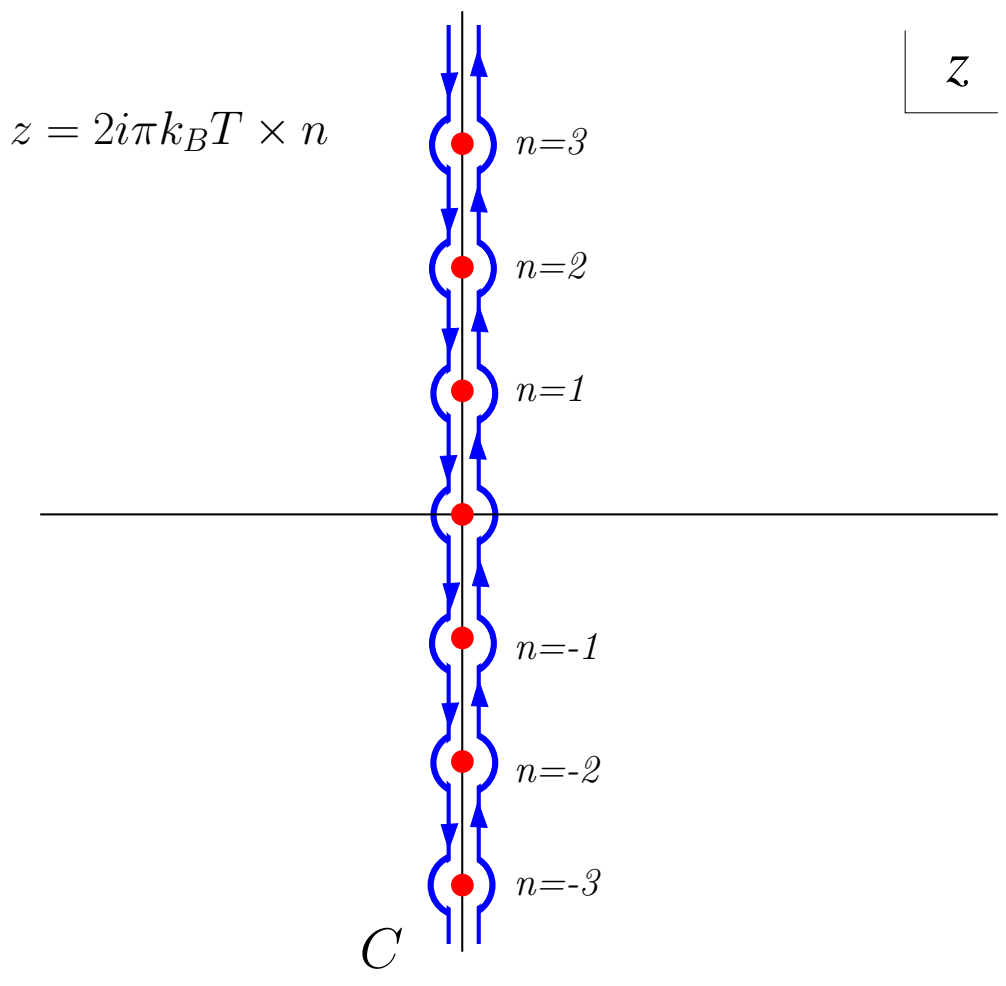

FIG. 2: Contour integration path $\mathcal{C}$ used to sum over the Matsubara frequencies.

By making this subtraction, we remove the ultraviolet divergences in the zero-point energy. By using the Matsubara method, we can reexpress the sum in Eq. (17) as

$$
\frac{1}{2 a c} \sum_{n} \sqrt{\left(c q_{\perp}\right)^{2}+\left(c q_{z n}\right)^{2}}=\frac{1}{\hbar c^{2} \beta_{C}} \sum_{n} F\left(i \nu_{n}\right),
$$

where

$$
F(z)=\sqrt{c^{2} q_{\perp}^{2}-z^{2}} .
$$

We associate the discrete wavevectors, $q_{z n}$, with a "Matsubara frequency" $c q_{z n} \equiv \nu_{n}$. Then

$$
c q_{z n}=c n \frac{\pi}{a} \equiv n\left(\frac{2 \pi k_{B} T_{C}}{\hbar}\right),
$$

where the effective Casimir temperature is given by

$$
k_{B} T_{C}=\frac{\hbar c}{2 a},
$$

so that

$$
\beta_{C}=\frac{1}{k_{B} T_{C}} \equiv \frac{2 a}{\hbar c} .
$$

We note that $T_{C}$ scales inversely with the plate separation $\left(T_{C} \sim 1 / 2 a\right)$. 
(a)

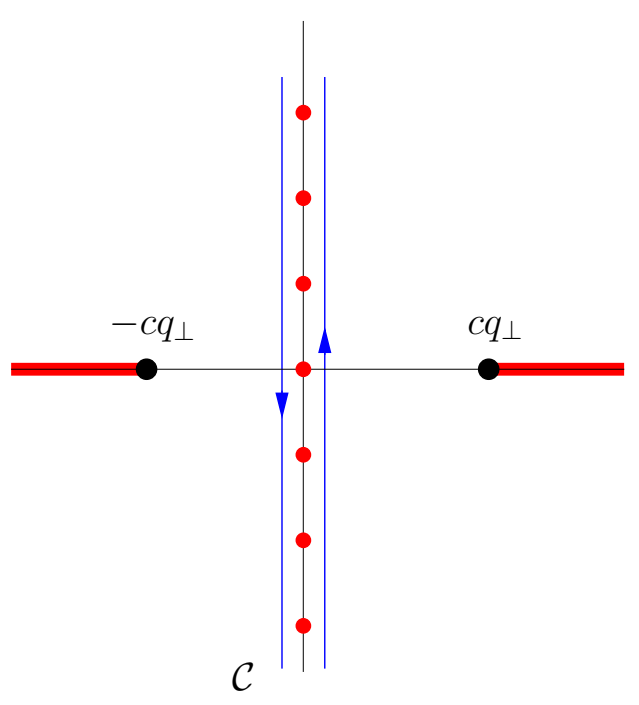

(b)

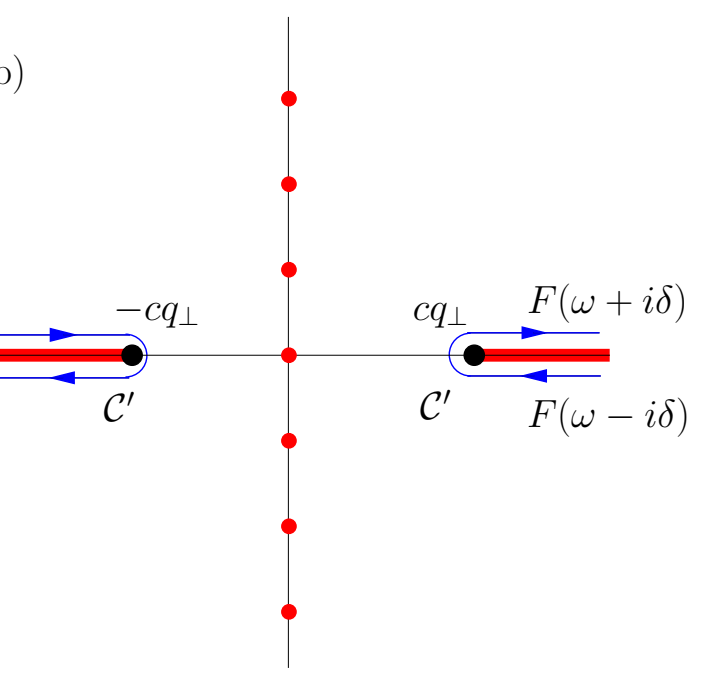

FIG. 3: (a) Contour integration path $\mathcal{C}$ used to calculate $\frac{1}{\hbar \beta_{C}} \sum_{n} F\left(i \nu_{n}\right)$ in Eq. (23), where $F(z)=\sqrt{c^{2} q_{\perp}^{2}-z^{2}}$, showing branch cuts in $F(z)$ at $z= \pm c q_{\perp}$. (b) Distortion of the contour into contour $C^{\prime}$ that wraps around the branch cuts of $F(z)$. The integrand of the integral along the branch cuts is the difference $F(\omega+i \delta)-F(\omega-i \delta)$ between the value of $F(z)$ above and below the branch cut.

Following the Matsubara approach, the sum in Eq. (18) can now be rewritten as a contour integral $^{22}$ around the poles at $z=i \nu_{n}$ of the Bose function $n_{B}\left(z, \beta_{C}\right)$ yielding

$$
\frac{1}{\hbar \beta_{C}} \sum_{n} F\left(i \nu_{n}\right)=\int_{C} \frac{d z}{2 \pi i} F(z) n_{B}\left(z, \beta_{C}\right) .
$$

The second term in $I\left(q_{\perp}, a\right)$, Eq. (17), corresponds to the $a \rightarrow \infty$, or $\beta_{C} \rightarrow \infty$ limit of the first term, and thus we may write

$$
I\left(q_{\perp}, a\right)=\lim _{\beta^{\prime} \rightarrow \infty}\left\{\frac{1}{c^{2}} \int_{C} \frac{d z}{2 \pi i} F(z)\left[n_{B}\left(z, \beta_{C}\right)-n_{B}\left(z, \beta^{\prime}\right)\right]\right\} .
$$

The subtraction of the $\beta_{C} \rightarrow \infty$ limit of the integrand in Eq. (24) regulates the overall integral at large $z$, guaranteeing that the integrand around a contour at infinity vanishes. This procedure permits us to evaluate the integral by distorting the contour around the branch cuts in $F(z)$ that extend from $z= \pm c q_{\perp}$ to infinity, as shown in Fig. (3)). We then obtain

$$
I\left(q_{\perp}, a\right)=\frac{1}{c^{2}}\left(\int_{-\infty}^{-c q_{\perp}}+\int_{c q_{\perp}}^{\infty}\right) \frac{d \omega}{2 \pi i}[F(\omega+i \delta)-F(\omega-i \delta)]\left[n_{B}\left(\omega, \beta_{C}\right)-\left\{\beta_{C} \rightarrow \infty\right\}\right] .
$$


To evaluate the branch cut, we note for $F(\omega \pm i \delta)=\sqrt{\left(c q_{\perp}\right)^{2}-(\omega \pm i \delta)^{2}}$,

$$
F(\omega+i \delta)-F(\omega-i \delta)=-2 i \sqrt{\omega^{2}-\left(c q_{\perp}\right)^{2}} \operatorname{sgn}(\omega)
$$

is an odd function of $\omega$, which permits us to replace $n_{B}(\omega)$ by its odd part $n_{B}(\omega)+\frac{1}{2}$ to obtain

$I\left(q_{\perp}, a\right)=\frac{1}{c^{2}}\left(\int_{-\infty}^{-c q_{\perp}}+\int_{c q_{\perp}}^{\infty}\right) \frac{d \omega}{2 \pi i}[F(\omega+i \delta)-F(\omega-i \delta)]\left[\left\{n_{B}\left(\omega, \beta_{C}\right)+\frac{1}{2}\right\}-\left\{\beta_{C} \rightarrow \infty\right\}\right]$.

Because the integrand is an even function of $\omega$, we can replace this integral by twice the integral over positive $\omega$ to obtain

$$
\begin{aligned}
I\left(q_{\perp}, a\right) & =-\frac{2}{c^{2}} \int_{c q_{\perp}}^{\infty} \frac{d \omega}{\pi} \sqrt{\omega^{2}-c^{2} q_{\perp}^{2}}\left[\left(n_{B}\left(\omega, \beta_{C}\right)+\frac{1}{2}\right)-\left\{\beta_{C} \rightarrow \infty\right\}\right] \\
& =-\frac{2}{c^{2}} \int_{c q_{\perp}}^{\infty} \frac{d \omega}{\pi} \sqrt{\omega^{2}-c^{2} q_{\perp}^{2}} n_{B}\left(\omega, \beta_{C}\right) .
\end{aligned}
$$

The change in zero point energy is then given by

$$
\frac{\Delta \mathcal{E}_{C}}{A}=-2 \hbar^{2} \beta_{C} \int_{\omega>q_{\perp}} \frac{d^{2} q_{\perp} d \omega}{(2 \pi)^{3}} n_{B}\left(\omega, \beta_{C}\right) \sqrt{\omega^{2}-c^{2} q_{\perp}^{2}},
$$

where we have made the substitution $2 a / c=\hbar \beta_{C}$. By carrying out the integral over $q_{\perp}$, we obtain

$$
\frac{\Delta \mathcal{E}_{C}}{A}=-\frac{\hbar^{2} \beta_{C}}{6 \pi^{2} c^{2}} \int d \omega \omega^{3} n_{B}\left(\omega, \beta_{C}\right)
$$

Rescaling the integral in Eq. (30) by changing variables to $x=\hbar \omega / k_{B} T$ and replacing $\beta_{C}=2 a / \hbar c$, we obtain

$$
\frac{\Delta \mathcal{E}_{C}}{A}=-\frac{1}{6 \pi^{2} \hbar^{2} \beta_{C}^{3} c^{2}} \overbrace{\int d x \frac{x^{3}}{e^{x}-1}}^{\frac{\pi^{4}}{15}}=-\frac{\pi^{2}}{720} \frac{\hbar c}{a^{3}}
$$

in numerical agreement with previous derivations. $1,17,18,19,20$ The associated force per unit area is then

$$
\frac{F}{A}=\frac{d \Delta \mathcal{E}_{c}}{d a}=\frac{\pi^{2}}{240} \frac{\hbar c}{a^{4}}=1.3 \times 10^{-3} \frac{1}{(a / \mu \mathrm{m})^{4}} \mathrm{~N} / \mathrm{m}^{2}
$$

indicating that measurements of the Casimir force must be performed at plate separations at or below the micrometer length scale. $\underline{5}^{6,7,8,9,10}$ When the two conducting plates are parallel, the force is attractive, but it can be repulsive in other situations. $\underline{24,25}$ 


\section{IMPLICATIONS}

We end with a discussion of the broader implications of our approach. Here we use the Bose function $n_{B}(\omega)$ to impose a spatial boundary condition on quantum fluctuations. Key to this treatment is the interpretation of the discrete $q_{z n}$ vectors as a set of Matsubara frequencies $c q_{z n} \equiv \nu_{n}$ so that we are effectively mapping the spatial frequencies of the Casimir effect to a discrete set of temporal frequencies in statistical mechanics. This procedure leads to an effective Casimir temperature Eq. (21),$k_{B} T_{C}=\hbar c / 2 a$, where $T_{C}$ is linked to a spatial boundary condition. If we now return to statistical mechanics, our treatment of the Casimir effect allows us to illustrate a deep relation between finite temperature and boundary conditions in time, not in space. This relation arises from the fact that the Boltzmann factor $e^{-\beta \hat{H}}$ corresponds to the unitary time evolution operator of quantum mechanics

$$
e^{-\beta \hat{H}}=e^{-\frac{i \hat{H}}{\hbar}[-i \hbar \beta]}=U[-i \hbar \beta]
$$

evaluated at the imaginary Planck time $t=-i \hbar \beta=-i \hbar / k_{B} T$. Thus statistical mechanics can be formulated as quantum mechanics in imaginary time, where the temporal evolution occurs along the imaginary time axis with $t=-i \tau$, where $\tau \in[0, \hbar \beta]$.

To give the reader a flavor for this link between finite temperature and temporal boundary conditions, we consider a simple harmonic oscillator described classically by the action

$$
S_{\mathrm{cl}}=\int_{t_{1}}^{t_{2}} d t\left[\frac{m \dot{\phi}^{2}}{2}-\frac{m \omega_{0}^{2} \phi^{2}}{2}\right]
$$

where $\phi(t), m$ and $\omega_{0}$ are its amplitude, mass, and angular frequency respectively. The passage from classical to quantum mechanics is achieved using Feynman's observation ${ }^{26}$ that the amplitude for the oscillator to follow a path $\phi(t)$ is $\exp \left[i S_{\mathrm{cl}} / \hbar\right]$. If $\left|\phi_{1}\right\rangle$ is the eigenstate of displacement, then the transition amplitude between $\left|\phi_{1}\right\rangle$ and $\left|\phi_{2}\right\rangle$ is the sum

$$
\left\langle\phi_{2}\left|U\left(t_{2}-t_{1}\right)\right| \phi_{1}\right\rangle=\sum_{\{\phi(t)\}} \exp \left[\frac{i}{\hbar} S_{\mathrm{cl}}\right] .
$$

over all paths that link $\phi_{1}$ and $\phi_{2}$. We pass from real to imaginary time, and identify $t \rightarrow-i \tau$

and the Euclidean action $S_{E}$ by $\frac{i}{\hbar} S_{\mathrm{cl}} \rightarrow-\frac{1}{\hbar} S_{E}$. By making the necessary replacements, we find that the imaginary time evolution from $\left|\phi_{1}\right\rangle$ to $\left|\phi_{2}\right\rangle$ is associated with the amplitude

$$
\left\langle\phi_{2}\left|e^{-\beta \hat{H}}\right| \phi_{1}\right\rangle=\sum_{\left\{\phi: \phi(0)=\phi_{1}, \phi(\hbar \beta)=\phi_{2}\right\}} \exp \left[-\frac{1}{\hbar} S_{E}\right] .
$$


To obtain the associated partition function, we must take the trace over this matrix

$$
\begin{aligned}
Z & =\operatorname{Tr}\left[e^{-\beta \hat{H}}\right]=\int_{-\infty}^{\infty} d \phi\left\langle\phi\left|e^{-\beta \hat{H}}\right| \phi\right\rangle=\int d \phi \sum_{\phi=\phi(0)=\phi(\hbar \beta)} \exp \left[-\frac{1}{\hbar} S_{E}\right] \\
& =\sum_{\phi(\hbar \beta)=\phi(0)} \exp \left(-\frac{1}{\hbar} S_{E}[\{\phi\}]\right),
\end{aligned}
$$

where the sum is over all periodic paths that satisfy $\phi(0)=\phi(\hbar \beta)$. We see that a finite temperature is formally equivalent to a periodic boundary condition in imaginary time. We next apply these ideas to our illustrative case.

In the passage from real to imaginary time, the quantum mechanical amplitude has been replaced by a "probability" $p[\{\phi\}] \propto e^{-S_{E}[\phi] / \hbar}$. To calculate this function for our simple example, we decompose the displacement in terms of its normal modes (Fig. 4) by Fourier transforming in imaginary time

$$
\phi(\tau)=\frac{1}{\sqrt{\hbar \beta}} \sum_{n} \phi_{n} e^{-i \nu_{n} \tau}
$$

where $\nu_{n}=2 \pi n / \hbar \beta$ and $\phi_{n}^{*}=\phi_{-n}$ because $\phi(\tau)=\phi(\tau)^{*}$ is real. We can then write the Euclidean action as a sum of contributions from each normal mode

$$
\frac{S_{E}[\{\phi\}]}{\hbar}=\frac{m}{2 \hbar} \sum_{n=-\infty}^{\infty}\left|\phi_{n}\right|^{2}\left[\omega_{0}^{2}+\nu_{n}^{2}\right]
$$

so that the probability amplitude $p[\{\phi\}]=Z^{-1} e^{-S_{E} / \hbar}$ factorizes into a product of Gaussian distribution functions for each normal mode:

$$
p[\{\phi\}] \propto \exp \left[-\sum_{n} \frac{\left|\phi_{n}\right|^{2}}{2 \sigma_{n}^{2}}\right]
$$

where

$$
\sigma_{n}^{2}=\left\langle\left|\phi_{n}\right|^{2}\right\rangle=\frac{\hbar}{m\left(\omega_{0}^{2}+\nu_{n}^{2}\right)}
$$

is the temperature-independent variance of each normal mode.

Let us now see how boundary conditions in time cause the system to become "hot" for our example. More specifically consider the variance in the displacement

$$
\left\langle\hat{\phi}^{2}\right\rangle=\frac{1}{\hbar \beta} \int_{0}^{\hbar \beta} d \tau\left\langle\hat{\phi}^{2}(\tau)\right\rangle=\frac{1}{\hbar \beta} \sum_{n}\left\langle\left|\phi_{n}\right|^{2}\right\rangle=k_{B} T \sum_{n} \frac{1}{m\left(\omega_{0}^{2}+\nu_{n}^{2}\right)},
$$

which has been rewritten in terms of the normal modes so that the average potential energy is given by

$$
\langle\hat{V}\rangle=\frac{m \omega_{0}^{2}}{2}\left\langle\phi^{2}\right\rangle=\frac{\omega_{0}^{2}}{2} k_{B} T \sum_{n} \frac{1}{\left(\omega_{0}^{2}+\nu_{n}^{2}\right)} .
$$




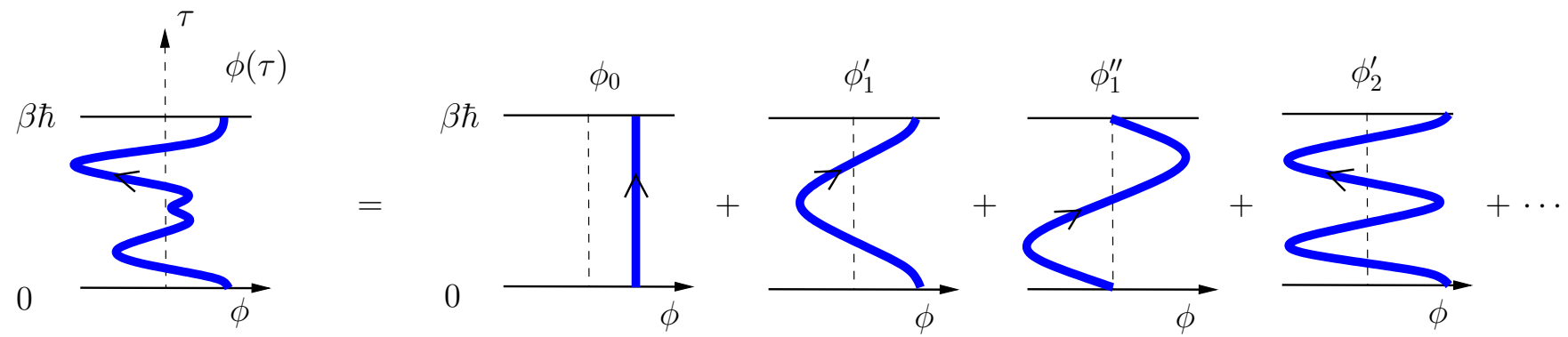

FIG. 4: Periodic paths in imaginary time for the harmonic oscillator can be decomposed into their Fourier modes, with amplitudes $\phi_{n}=\phi_{n}^{\prime}+i \phi_{n}^{\prime \prime}$.

(a)

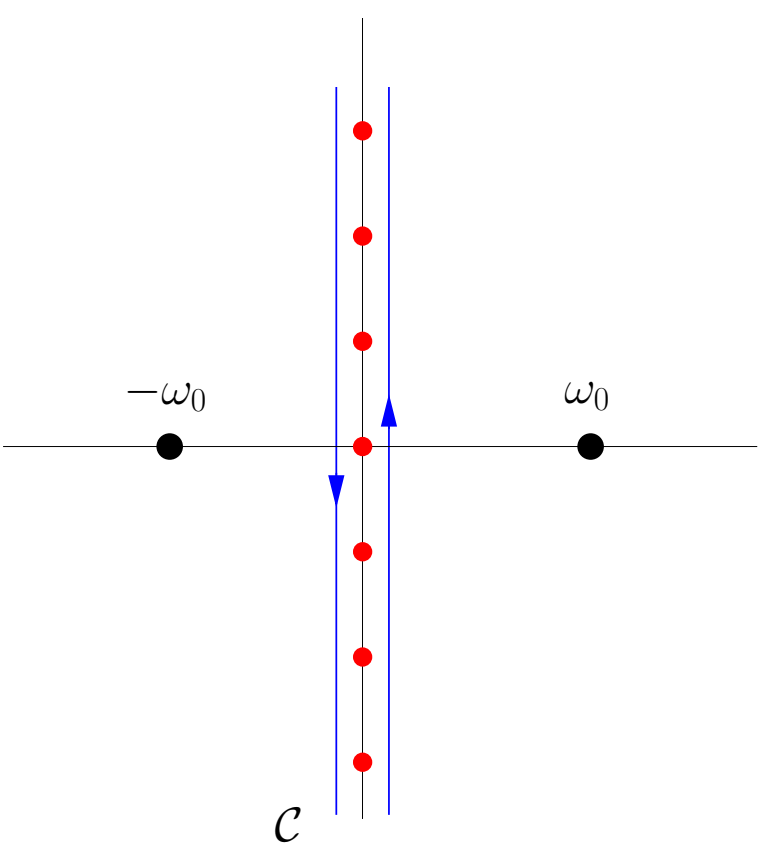

(b)

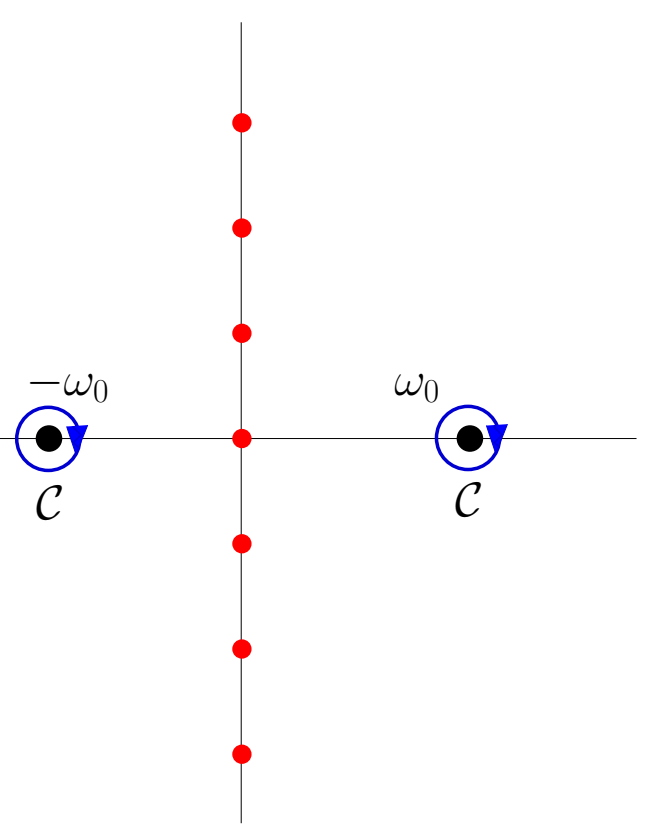

FIG. 5: (a) The contour integration path $\mathcal{C}$ used to evaluate Eq. (45). (b) The distortion of the contour to form the path $\mathcal{C}^{\prime}$ around the poles at $z= \pm \omega_{0}$.

We can calculate this expression using contour integration methods. We rewrite it as a clockwise contour integral around the poles of $n(z)=\left[e^{\hbar z / k_{B} T}-1\right]^{-1}$ at $z=i \nu_{n}$ (Fig. [5(a))

$$
\begin{aligned}
\langle\hat{V}\rangle & =\frac{\hbar \omega_{0}^{2}}{2} \oint_{C} \frac{d z}{2 \pi i} n_{B}(z) \frac{1}{\left(\omega_{0}^{2}-z^{2}\right)} \\
& =\frac{\hbar \omega_{0}^{2}}{2} \oint_{C^{\prime}} \frac{d z}{2 \pi i} n_{B}(z) \frac{1}{\left(\omega_{0}^{2}-z^{2}\right)}
\end{aligned}
$$

where $C^{\prime}$ runs clockwise around the poles at $z= \pm \omega_{0}$ (Fig. 5(b)). The resulting integral is 


$$
\begin{aligned}
\langle\hat{V}\rangle & =\frac{\hbar \omega_{0}^{2}}{2}\left(\frac{n_{B}\left(\omega_{0}\right)}{2 \omega_{0}}+\frac{n_{B}\left(-\omega_{0}\right)}{-2 \omega_{0}}\right) \\
& =\frac{\hbar \omega_{0}}{2}\left[n_{B}\left(\omega_{0}\right)+\frac{1}{2}\right] .
\end{aligned}
$$

From the virial theorem ${ }^{27}$ we expect a similar expression for the kinetic energy so that the total energy of the harmonic oscillator is

$$
\langle\hat{T}+\hat{V}\rangle=\hbar \omega_{0}\left[n_{B}\left(\omega_{0}\right)+\frac{1}{2}\right] .
$$

The second term is due to $T=0$ zero-point fluctuations, and the first describes the thermal excitations of the oscillator. From this derivation we see that the latter result from the redistribution of normal mode energies, and are a direct consequence of the imposition of a boundary condition in (imaginary) time.

Blackbody radiation is a well-known example of thermally excited oscillators. We can easily generalize Eq. (46) to the electromagnetic vacuum, an ensemble of harmonic oscillators, by replacing $\omega_{0} \rightarrow c q$, so that the energy density at a finite temperature is given by

$$
\begin{aligned}
\frac{\mathcal{E}_{\mathcal{T}}}{V} & =\frac{1}{V} \sum_{\mathbf{q}} 2 \hbar c q\left[n_{B}(c q)+\frac{1}{2}\right] \\
& =2 \int \frac{d^{3} q}{(2 \pi)^{3}} \hbar c q\left[n_{B}(c q)+\frac{1}{2}\right],
\end{aligned}
$$

where the factor of two is due to the different polarizations. With the substitution $x=\hbar c q \beta$, the thermal energy density takes the form

$$
\frac{\Delta \mathcal{E}_{T}}{V}=\frac{\left(k_{B} T\right)^{4}}{\pi^{2} c^{3} \hbar^{3}} \int d x x^{3} n_{B}(x)
$$

We compare Eq. (48) with the energy density obtained in the Casimir effect in Eq. (30). With $\beta_{C}^{-1}=k_{B} T_{C}=\hbar c / 2 a$, the Casimir energy density becomes

$$
\frac{\Delta \mathcal{E}_{C}}{A a}=-\frac{\left(k_{B} T_{C}\right)^{4}}{3 \pi^{2} c^{3} \hbar^{3}} \int d x x^{3} n_{B}(x) .
$$

The similarity between the Casimir and the blackbody energy density is testament to their common origin as boundary-condition effects. The sign difference is due to the subtle distinctions between imaginary and real time, and the consequences for the kinetic energy. As an aside, we note that the blackbody radiation pressure, $P=\Delta \mathcal{E}_{T} / 3 V$, has the same pref-

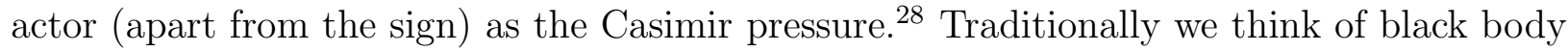


radiation as resulting from an excitation of thermal modes. Our calculation shows that the Casimir effect and blackbody radiation are both consequences of boundary conditions and the redistribution of zero-point fluctuation modes in the vacuum.

Recent experiments have observed the Casimir effect between parallel plates with a one micron separation $(a=1 \mu \mathrm{m}), \underline{5,6,7,8,9,10}$ The corresponding "Casimir temperature" for these experiments is

$$
T_{C}=\frac{\hbar c}{2 a k_{B}} \sim 1000 \mathrm{~K}
$$

The Casimir effect at these length scales couples to the same photons that predominate in the blackbody spectrum at $1000 \mathrm{~K}$. The boundary conditions imposed by the two phenomena on the electromagnetic field are almost identical.

More generally, zero-point fluctuations play a major role at quantum phase transitions. $.30,31,32$ The effect of finite temperature in the vicinity of a $(T=0)$ quantum critical point is the temporal analog of the Casimir phenomenon, a "Casimir effect in time," where temperature imposes temporal constraints on critical zero-point fluctuations. As we have discussed, there is an intimate connection between a finite temporal dimension and a nonzero temperature in a quantum system, $, 29,30,31,32,33$ and this connection has many observable consequences on thermodynamic quantities for quantum critical systems at nonzero temperatures. ${ }^{16}$ Heuristically, this link between temperature and a boundary condition in time can be understood within the framework of the Heisenberg uncertainty principle

$$
\Delta t \sim \frac{\hbar}{k_{B} T}
$$

where a thermal energy fluctuation leads to an upper cutoff in time, the Planck time, that is inversely proportional to the temperature. More formally, as we have seen in our simple example, finite-temperature emerges in a path integral framework as a periodic boundary effect in imaginary time, which becomes particularly important near a quantum critical point where there exist quantum fluctuations on all spatial and temporal scales. Here finitetemperature corresponds to the redistribution of quantum zero-point fluctuations due to the imposition of external constraints, and thus is analogous to the Casimir effect for two parallel metallic plates in vacuum. Running this argument the other way, we note that the removal of temporal modes by periodic finite boundary conditions generates a temperature (and thus entropy and thermal energy) in a system near a quantum critical point. As an aside, we remark that finite-temperature effects resulting from boundary constraints have 
been discussed in the context of astrophysics where blackbody radiation and event horizons have been linked via the Unruh effect. $\underline{\underline{34}, 35}$

In conclusion, we have revisited the Casimir effect with an approach used to study condensed matter systems at finite temperature. We recovered results previously derived by other methods and also discussed the physical implications of analogies implicit in this treatment. Our hope is that this presentation will make this topic straightforward to include in a graduate many-body course for future condensed matter physicists.

\section{Acknowledgments}

This perspective on the Casimir effect emerged from research on quantum paraelectrics, funded by the DOE (grant DE-FE02-00ER45790), and research on finite-size phenomena in quantum systems, supported by the NSF (grants nsf-dmr 0645461 and NSF-NIRT-ECS0608842).

1 H. B. G. Casimir, "On the attraction between two perfectly conducting plates," Proc. Kon. Ned. Akad. Wetenschap 51, 793-795 (1948).

2 A. Lambrecht, "The Casimir effect: A force from nothing," Physics World 15 (9), 29-32 (2002).

3 S. K. Lamoroeaux, "Casimir forces: Still surprising after 60 years," Phys. Today 60 (2), 40-45 (2007).

4 C. Itzykson and J.-B. Zuber, Quantum Field Theory (McGraw-Hill, New York, 1980).

5 S. K. Lamoreaux, "Demonstration of the Casimir force on the 0.6 to $6 \mu \mathrm{mm}$ range," Phys. Rev. Lett. 78, 5-8 (1997).

6 U. Mohideen and A. Roy, "Precision measurement of the Casimir force from 0.1 to $0.9 \mu \mathrm{m}, "$ Phys. Rev. Lett. 81, 4549-4552 (1998).

7 H. B. Chan, V. A. Aksyuk, R. N. Kleiman, D. J. Bishop, and F. Capasso, "Quantum mechanical actuation of micromechanical systems by the Casimir force," Science 291, 1941-1944 (2001).

8 M. Lisanti, D. Iannuzzi, and F. Capasso, "Observation of the skin-depth effect on the Casimir force between metallic surfaces," PNAS 102, 11989-11992 (2005).

9 J. M. Obrecht, R. J. Wild, M. Antezza, L. P. Pitaevskii, S. Stringari, and E. A. Cornell, 
"Measurement of the temperature-dependence of the Casimir-Polder force," Phys. Rev. Lett. 98, 063201-1-4 (2007).

10 J. N. Munday, F. Capasso, and V. A. Parsegian, "Measured long-range repulsive Casimir-Lifshitz forces," Nature 457, 170-173 (2009).

11 M. E. Fisher and P. G. deGennes, C.R. Acad. Sci. Paris B 287, 207-209 (1978).

12 M. Krech, The Casimir Effect in Critical Systems (World Scientific, Singapore, 1994).

13 M. Kardar and R. Golestanian, "The 'friction' of vacuum, and other fluctation-induced forces," Rev. Mod. Phys. 71, 1233-1245 (1999).

14 D. M. Danchev, J. G. Brankov and N. S. Tonchev, Theory of Critical Phenomena in Finite-Size Systems: Scaling and Quantum Effects (World Scientific, Singapore, 2000).

15 A. Gambassi, "The Casimir effect: From quantum to critical fluctuations," J. Phys.: Conf. Ser. 161 012037-1-17 (2009).

16 L. Pálová, P. Chandra, and P. Coleman, "Quantum critical paraelectrics and the Casimir effect in time," Phys. Rev. B 79, 075101-1-17 (2009).

17 F. J. Belinfante, "The Casimir effect revisited," Am. J. Phys. 55, 134-138 (1987).

18 J. P. Dowling, "The mathematics of the Casimir effect," Mathematics Magazine 62, 324-331 (1989).

19 E. Elizalde and A. Romeo, "Essentials of the Casimir effect and its computation," Am. J. Phys. 59, 711-719 (1991).

20 M. Revzen, R. Opher, M. Opher, and A. Mann, "Kirchoff's theorem and the Casimir effect," Europhys. Lett. 38, 245-248 (1997).

21 S. K. Lamoreaux, "Resource Letter CF-1: Casimir force," Am. J. Phys. 67, 850-861 (1999).

22 G. D. Mahan, Many-Particle Physics (Springer, New York, 2007), 3rd ed.

23 J. D. Jackson, Classical Electrodynamics (John Wiley \& Sons, New York, 1998), 3rd ed., pp. $352-363$.

24 T. H. Boyer, "Van der Waals forces and zero-point entropy for dielectric and permeable materials," Phys. Rev. A 9, 2078-2084 (1974).

25 V. Hushwater, "Repulsive Casimir force as a result of vacuum radiation pressure," Am. J. Phys. 65, 381-384 (1997).

26 R. P. Feynman and A. R. Hibbs, Quantum Mechanics and Path Integrals (McGraw-Hill, New York, 1965); R. P. Feynman, "Space-time approach to non-relativistic quantum mechanics," 
Rev. Mod. Phys. 20, 367-387 (1948).

27 R. K. Pathria, Statistical Mechanics (Butterworth-Heinemann, Oxford, 1996), 2nd ed.

28 L. D. Landau and E. M. Lifshitz, Statistical Physics (Pergamon Press, Oxford, 1980).

29 J. Cardy, Scaling and Renormalization in Statistical Physics (Cambridge University Press, Cambridge, 1996).

30 S. L. Sondhi, S. M. Girvan, J. P. Carini, and D. Shahar, "Continuous quantum phase transitions," Rev. Mod. Phys. 69, 315-333 (1997).

31 S. Sachdev, Quantum Phase Transitions (Cambridge University Press, Cambridge, 1999).

32 P. Coleman and A. J. Schofield, "Quantum criticality," Nature 433, 226-229 (2005).

33 M. A. Continentino, Quantum Scaling in Many-Body Systems (World Scientific, Singapore, 2001).

34 W. G. Unruh, "Notes on black hole evaporation," Phys. Rev. D 14, 870-892 (1976).

35 K. Thorne, Black Holes and Time Warps: Einstein's Outgrageous Legacy (W. W. Norton, New York, 1995). 